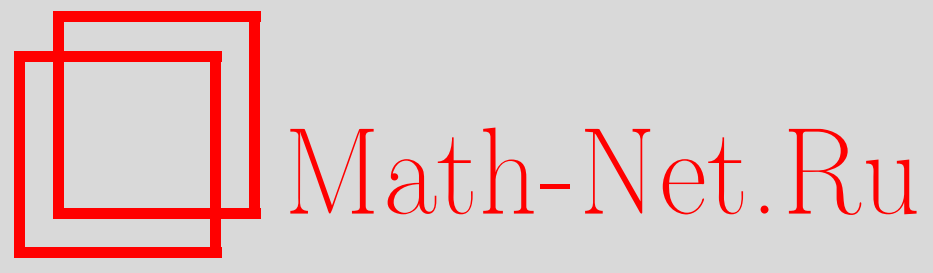

О. А. Репин, С. К. Кумыкова, Задача со смещением для вырождающегося внутри области гиперболического уравнения, Вестн. Сам. гос. техн. ун-та. Сер. Физ.-мат. науки, 2014, выпуск 1(), 37-47

DOI: https://doi.org/10.14498/vsgtu1280

Использование Общероссийского математического портала MathNet.Ru подразумевает, что вы прочитали и согласны с пользовательским соглашением

http://www.mathnet.ru/rus/agreement

Параметры загрузки:

IP: 3.85 .7 .115

26 апреля 2023 г., 13:19:56

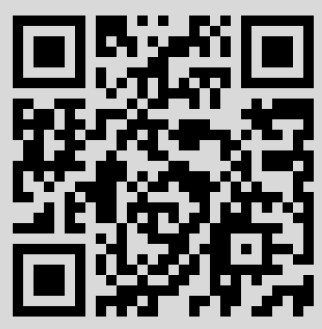


Вестн. Сам. гос. техн. ун-та. Сер. Физ.-мат. науки. 2014. № 1 (34). С. $37-47$

УДК 517.956.326

\title{
ЗАДАЧА СО СМЕЩЕНИЕМ ДЛЯ ВЫРОЖДАЮЩЕГОСЯ ВНУТРИ ОБЛАСТИ ГИПЕРБОЛИЧЕСКОГО УРАВНЕНИЯ
}

\author{
O. А. Репин ${ }^{1,2}$, C. К. Кумыкова \\ 1 Самарский государственный экономический университет, \\ Россия, 443090, Самара, ул. Советской Армии, 141. \\ 2 Самарский государственный технический университет, \\ Россия, 443100, Самара, ул. Молодогвардейская, 244. \\ 3 Кабардино-Балкарский государственный университет им. Х. М. Бербекова \\ Россия, 360004, Нальчик, ул. Чернышевского, 173.
}

\begin{abstract}
Для вырождающегося гиперболического уравнения в характеристической области (двуугольнике) исследована внутреннекраевая задача с операторами дробного интегро-дифферениирования (в смысле Римана-Лиувилля), в которой значения решения уравнения на характеристиках поточечно связаны со значением решения и производной от него на линии вырождения уравнения. Модифицированным методом Трикоми при ограничениях в виде неравенств на известные бункиии доказана теорема единственности. Вопрос существования решения задачи редуцирован $к$ разрешимости сингулярного интегрального уравнения с ядром Коши нормального типа.
\end{abstract}

Ключевые слова: задача Коши, задача со смещением, операторы дробного интегро-дифференцирования, сингулярное уравнение с ядром Коши, регуляризатор, гипергеометрическая функиия Гаусса, гамма-функиия Эйлера.

\section{1. Постановка задачи. Рассмотрим уравнение}

$$
|y|^{m} u_{x x}-u_{y y}+a|y|^{m / 2-1} u_{x}=0,
$$

где $m=$ const $>2 ; a \neq 0$ - действительная постоянная, $|a| \leqslant m / 2$ в конечной области $\Omega$, ограниченной характеристиками

$$
\begin{gathered}
A C: x-\frac{2}{m+2} y^{(m+2) / 2}=0, \quad B C: x+\frac{2}{m+2} y^{(m+2) / 2}=1, \\
A D: x-\frac{2}{m+2}(-y)^{(m+2) / 2}=0, \quad B D: x+\frac{2}{m+2}(-y)^{(m+2) / 2}=1 .
\end{gathered}
$$

Пусть $\Omega_{1}=\Omega \cap(y>0), \Omega_{2}=\Omega \cap(y<0), I \equiv A B$ - единичный интервал $0<x<1$ прямой $y=0 ;\left(I_{0+}^{\alpha} f\right)(x),\left(I_{1-}^{\alpha} f\right)(x),\left(D_{0+}^{\alpha} f\right)(x),\left(D_{1-}^{\alpha} f\right)(x)-$ операторы дробного интегро-дифференцирования в смысле Римана-Лиувилля [1, с. 42-43]; $\Theta_{0}^{i}(x), \Theta_{1}^{i}(x)$ - точки пересечения характеристик уравнения

ISSN: 2310-7081 (online), 1991-8615 (print); doi: http://dx.doi.org/10.14498/vsgtu1280 (C) 2014 Самарский государственный технический университет.

Образец цитирования: О. А. Р епи и, С. К. Кумы ко в а, “Задача со смещением для вырождающегося внутри области гиперболического уравнения" // Вестн. Сам. гос. техн. ун-та. Сер. Физ.-мат. науки, 2014. № 1 (34). С. 37-47. doi: 10.14498/vsgtu1280.

Сведения об авторах: Олег Александрович Репин (д.ф.-м.н., проф.), заведующий кафедрой, каф. математической статистики и эконометрики ${ }^{1}$; профессор, каф. прикладной математики и информатики ${ }^{2}$. Светлана Каншубиевна Кумыкова (к.ф.-м.н., доц.), доцент, каф. теории функций и функционального анализа.

E-mail addresses: matstat@mail.ru (O.A. Repin, Corresponding author), bsk@rect.kbsu.ru (S.K. Kumykova) 
$(1)$, выходящих из точки $(x, 0) \in I$, с характеристиками $A C, B C, A D, B D$ соответственно, $i=1,2$.

ЗАДАчА. Найти решение $u(x, y)$ уравнения (1) со свойствами

1) $u(x, y) \in C(\bar{\Omega}) \cap C^{1}\left(\Omega_{1} \cup I\right) \cap C^{1}\left(\Omega_{2} \cup I\right) \cap C^{2}\left(\Omega_{1} \cup \Omega_{2}\right)$;

2) $u(x,+0)=u(x,-0), x \in \bar{I}$;

$\lim _{y \rightarrow+0} u_{y}(x, y)=\mu(x) \lim _{y \rightarrow-0} u_{y}(x, y)+\lambda(x), x \in I$

3) $A_{i}(x) I_{0+}^{p_{i}} \delta_{i}(x) u\left[\Theta_{0}^{i}(x)\right]+B_{i}(x) I_{1-}^{q_{i}} w_{i}(x) u\left[\Theta_{1}^{i}(x)\right]+$

$+C_{i}(x) u_{i y}(x, 0)+D_{i}(x) u_{i}(x, 0)=\gamma_{i}(x), \quad i=1,2, x \in I$, где $A_{i}(x), B_{i}(x), C_{i}(x), D_{i}(x) \gamma_{i}(x), \delta_{i}(x), w_{i}(x), \mu(x), \lambda(x)$ - заданнье функиии такие, что

$$
\begin{aligned}
& A_{i}^{2}(x)+B_{i}^{2}(x)+C_{i}^{2}(x)+D_{i}^{2}(x) \neq 0, \\
& A_{i}(x), B_{i}(x), C_{i}(x), D_{i}(x), \gamma_{i}(x) \in C^{1}(\bar{I}) \cap C^{3}(I), \quad \mu(x), \lambda(x) \in C^{1}(I), \\
& p_{i}, q_{i} \text {-действительные постоянные, причём } 0<p_{i}, q_{i}<1 .
\end{aligned}
$$

Отметим, что рассматриваемая задача относится к классу краевых задач со смещением [2] (по терминологии А. М. Нахушева).

\section{2. Единственность решения задачи.}

Теорема. В области $\Omega$ не может существовать более одного решения задачи, если

$$
\begin{gathered}
\mu(x)=1, \quad \lambda(x)=0, \quad p_{1}=p_{2}=\alpha, \quad q_{1}=q_{2}=\beta, \\
\delta_{1}(x)=\delta_{2}(x)=x^{\alpha+\beta-1}, \quad w_{1}(x)=w_{2}(x)=(1-x)^{\alpha+\beta-1} ; \\
E_{i}(x)=\frac{\Gamma(\alpha+\beta)}{\Gamma(\beta)}(1-x)^{1-\alpha} A_{i}(x)+\frac{\Gamma(\alpha+\beta)}{\Gamma(\alpha)} x^{1-\beta} B_{i}(x)+ \\
+x^{1-\beta}(1-x)^{1-\alpha} D_{i}(x) \neq 0, \quad i=1,2, x \in \bar{I}
\end{gathered}
$$

и выполняются неравенства

$$
\begin{gathered}
{\left[\frac{A_{1}(x)}{E_{1}(x)}(1-x)^{1-\alpha}\right]^{\prime} \leqslant 0,\left[\frac{B_{1}(x)}{E_{1}(x)} x^{1-\beta}\right]^{\prime} \geqslant 0, \frac{C_{1}(x)}{E_{1}(x)} x^{1-\beta}(1-x)^{1-\alpha} \leqslant 0,} \\
{\left[\frac{A_{2}(x)}{E_{2}(x)}(1-x)^{1-\alpha}\right]^{\prime} \geqslant 0,\left[\frac{B_{2}(x)}{E_{2}(x)} x^{1-\beta}\right]^{\prime} \leqslant 0, \frac{C_{2}(x)}{E_{2}(x)} x^{1-\beta}(1-x)^{1-\alpha} \geqslant 0, x \in \bar{I},}
\end{gathered}
$$

либо

$$
\begin{aligned}
& \mu(x)>0, \quad \lambda(x)=0, \quad p_{1}=p_{2}=1-\beta \\
& q_{1}=q_{2}=1-\alpha, \quad \delta(x)=w(x)=1
\end{aligned}
$$

$$
\begin{aligned}
\widetilde{E}_{i}(x)=\frac{\Gamma(2-\alpha-\beta)}{\Gamma(1-\alpha)}\left(\frac{m+2}{4}\right)^{2 /(m+2)}(1-x)^{\beta} A_{i}(x)+ & \\
+\frac{\Gamma(2-\alpha-\beta)}{\Gamma(1-\beta)}\left(\frac{m+2}{4}\right)^{2 /(m+2)} x^{\alpha} B_{i}(x)+ & \\
& +x^{\alpha}(1-x)^{\beta} C_{i}(x) \neq 0, \quad i=1,2, x \in \bar{I}
\end{aligned}
$$


и выполняются неравенства

$$
\begin{gathered}
\frac{A_{1}(x)}{\widetilde{E}_{1}(x)}(1-x)^{\beta}<0, \frac{B_{1}(x)}{\widetilde{E}_{1}(x)} x^{\alpha}<0, \frac{D_{1}(x)}{\widetilde{E}_{1}(x)} x^{\alpha}(1-x)^{\beta}<0, \\
\frac{A_{2}(x)}{\widetilde{E}_{2}(x)}(1-x)^{\beta}>0, \frac{B_{2}(x)}{\widetilde{E}_{2}(x)} x^{\alpha}>0, \frac{D_{2}(x)}{\widetilde{E}_{2}(x)} x^{\alpha}(1-x)^{\beta}>0, \quad x \in \bar{I},
\end{gathered}
$$

либо

$$
p_{1}=\alpha, \quad p_{2}=1-\beta, \quad q_{1}=\beta, \quad q_{2}=1-\alpha, \quad \delta(x)=w(x)=1 ;
$$

выполняются условия (4), (5) в области $\Omega_{1}$, а в области $\Omega_{2}$ справедливы условия (7), (9), где

$$
\alpha=\frac{m-2 a}{2(m+2)}, \quad \beta=\frac{m+2 a}{2(m+2)} .
$$

Доказ ательство. Переходя к доказательству единственности решения задачи, положим

$$
\tau(x)=u(x, 0), \quad \nu_{1}(x)=\lim _{y \rightarrow 0+0} u_{y}(x, y), \quad \nu_{2}(x)=\lim _{y \rightarrow 0-0} u_{y}(x, y) .
$$

Пусть выполняются условия (3), (4) теоремы.

Используя формулу решения задачи Коши для уравнения (1) в областях $\Omega_{1}, \Omega_{2}$ [3, с. 13-14] и удовлетворяя краевым условиям 3$)$, получим соотношения между $\tau(x)$ и $\nu_{i}(x)$, принесённые на $I$ из $\Omega_{1}$ и $\Omega_{2}$ соответственно:

$$
\tau(x)=\bar{A}_{i}(x) I_{0+}^{1-\alpha-\beta} \nu_{i}(x)+\bar{B}_{i}(x) I_{1-}^{1-\alpha-\beta} \nu_{i}(x)+\bar{C}_{i}(x) \nu_{i}(x)+\bar{\gamma}_{i}(x),
$$

где

$$
\begin{gathered}
\bar{A}_{i}(x)=-\frac{\Gamma(2-\alpha-\beta)}{\Gamma(1-\alpha)}\left(\frac{m+2}{4}\right)^{2 /(m+2)} \frac{A_{i}(x)}{E_{i}(x)}(1-x)^{1-\alpha}, \\
\bar{B}_{i}(x)=-\frac{\Gamma(2-\alpha-\beta)}{\Gamma(1-\beta)}\left(\frac{m+2}{4}\right)^{2 /(m+2)} \frac{B_{i}(x)}{E_{i}(x)} x^{1-\beta}, \\
\bar{C}_{i}(x)=-\frac{C_{i}(x)}{E_{i}(x)} x^{1-\beta}(1-x)^{1-\alpha}, \\
\bar{\gamma}_{i}(x)=\frac{\gamma_{i}(x)}{E_{i}(x)} x^{1-\beta}(1-x)^{1-\alpha}, \quad i=1,2 .
\end{gathered}
$$

После преобразований, аналогичных [4,5], получим, что

$$
\int_{0}^{1} \tau(x) \nu_{i}(x) d x=0 .
$$

Затем нетрудно доказать равенство $\nu_{i}(x)=0$ (см., например, [4]). Тогда из (11) при $\widetilde{\gamma}_{i}(x)=0$ имеем $\tau(x)=0$. Следовательно, $u_{i}(x, y) \equiv 0$ как решения задачи Коши с нулевыми данными в областях $\Omega_{1}, \Omega_{2}$, что и завершает 
доказательство единственности решения исследуемой задачи для уравнения (1).

Если выполняются условия (6), (7) теоремы, то соотношения между $\tau(x)$ и $\nu_{i}(x)$ имеют вид

$$
\nu_{i}(x)=\widetilde{A}_{i}(x) D_{0+}^{1-\alpha-\beta} \tau(x)+\widetilde{B}_{i}(x) D_{1-}^{1-\alpha-\beta} \tau(x)+\widetilde{D}_{i}(x) \tau(x)+\widetilde{\gamma}_{i}(x),
$$

где

$$
\begin{aligned}
& \widetilde{A}_{i}(x)=-\frac{\Gamma(\alpha+\beta)}{\Gamma(\beta)} \frac{A_{i}(x)}{\widetilde{E}_{i}(x)}(1-x)^{\beta}, \quad \widetilde{B}_{i}(x)=-\frac{\Gamma(\alpha+\beta)}{\Gamma(\alpha)} \frac{B_{i}(x)}{\widetilde{E}_{i}(x)} x^{\alpha} \\
& \widetilde{D}_{i}(x)=-\frac{D_{i}(x)}{\widetilde{E}_{i}(x)} x^{\alpha}(1-x)^{\beta}, \quad \widetilde{\gamma}_{i}(x)=\frac{\gamma_{i}(x)}{\widetilde{E}_{i}(x)} x^{\alpha}(1-x)^{\beta}, \quad i=1,2 .
\end{aligned}
$$

В силу принципа экстремума для гиперболических уравнений положительный максимум (отрицательный минимум) функции $u(x, y)$ в $\bar{\Omega}_{1}, \bar{\Omega}_{2}$ достигается на $\bar{I}$. Пусть положительный максимум функции $u(x, y)$ достигается в точке $\left(x_{0}, 0\right) \in I$.

Пользуясь тем, что дробные производные $D_{0+}^{1-\alpha-\beta} \tau(x), D_{1-}^{1-\alpha-\beta} \tau(x)$ в точке положительного максимума строго положительны (в точке отрицательного минимума строго отрицательны) [2, с. 82-83], получаем при выполнении условий $(8),(9) \nu_{1}\left(x_{0}\right)>0, \nu_{2}\left(x_{0}\right)<0$.

Это противоречит условию сопряжения 2) при $\mu(x)>0, \lambda(x)=0$, откуда и следует справедливость теоремы единственности решения рассматриваемой задачи для уравнения (1).

3. Существование решения задачи. До ка з а $m$ ел з с $m$ в о существования решения задачи проведём для трёх случаев.

СлучАй 1. Пусть в областях $\Omega_{1}$ и $\Omega_{2}$ выполняются условия (3) и

$$
C_{2}(x) E_{1}(x)-C_{1}(x) E_{2}(x)=0 .
$$

Полагая $\nu_{1}(x)=\nu_{2}(x)=\nu(x)$ и удовлетворяя (11), требованию сопряжения 2), получим

$$
A(x) I_{0+}^{1-\alpha-\beta} \nu(x)+B(x) I_{1-}^{1-\alpha-\beta} \nu(x)=F(x),
$$

где

$$
A(x)=\bar{A}_{1}(x)-\bar{A}_{2}(x), \quad B(x)=\bar{B}_{1}(x)-\bar{B}_{2}(x), \quad F(x)=\bar{\gamma}_{2}(x)-\bar{\gamma}_{1}(x) .
$$

Здесь $m>2,0<\alpha, \beta<1, \alpha+\beta=m /(m+2)$.

Разделим обе части $(13)$ на $A(x) \neq 0$, а затем к обеим частям получившегося соотношения применим оператор $D_{0+}^{1-\alpha-\beta}$.

В результате будем иметь

$$
\nu(x)+D_{0+}^{1-\alpha-\beta} M(x) I_{1-}^{1-\alpha-\beta} \nu(x)=D_{0+}^{1-\alpha-\beta}\left[\frac{F(x)}{A(x)}\right],
$$

где $M(x)=B(x) / A(x)$. 
Используя методику и результаты работы [6], а также монографии [7, с. 81-89], можно записать

$$
I_{1}(x)=D_{0+}^{1-\alpha-\beta} M(x) I_{1-}^{1-\alpha-\beta} \nu(x)
$$

в виде

$$
I_{1}(x)=\cos [\pi(\alpha+\beta)] M(x) \nu(x)+\mu \int_{0}^{x} K_{1}(x, \xi) \nu(\xi) d \xi+\mu \int_{x}^{1} K_{2}(x, \xi) \nu(\xi) d \xi
$$

где

$$
\begin{aligned}
& \mu=\frac{1}{\Gamma(\alpha+\beta) \Gamma(1-\alpha-\beta)}=\frac{\sin [\pi(\alpha+\beta)]}{\pi} \\
& K_{1}(x, \xi)=\frac{d}{d x} \int_{0}^{\xi} \frac{M(t) d t}{(x-t)^{1-\alpha-\beta}(\xi-t)^{\alpha+\beta}} \\
& K_{2}(x, \xi)=\frac{d}{d x} \int_{0}^{x} \frac{M(t) d t}{(x-t)^{1-\alpha-\beta}(\xi-t)^{\alpha+\beta}} .
\end{aligned}
$$

Исследуем поведение ядер $K_{1}(x, \xi)$ и $K_{2}(x, \xi)$. Имеем

$$
\begin{aligned}
K_{1}(x, \xi)=M(\xi) \frac{d}{d x} \int_{0}^{\xi} \frac{d t}{(x-t)^{1-\alpha-\beta}(\xi-t)^{\alpha+\beta}}- \\
-\frac{d}{d x} \int_{0}^{\xi} \frac{[M(\xi)-M(t)] d t}{(x-t)^{1-\alpha-\beta}(\xi-t)^{\alpha+\beta}} .
\end{aligned}
$$

Гладкость ядра $K_{1}(x, \xi)$ определяется гладкостью первого интеграла

$$
\begin{aligned}
I_{2}(x, \xi) & =M(\xi) \frac{d}{d x} \int_{0}^{\xi} \frac{d t}{(x-t)^{1-\alpha-\beta}(\xi-t)^{\alpha+\beta}}= \\
& =\frac{M(\xi)}{1-\alpha-\beta} \frac{d}{d x}\left[\left(\frac{\xi}{x}\right)^{1-\alpha-\beta} F\left(1-\alpha-\beta, 1 ; 2-\alpha-\beta ; \frac{\xi}{x}\right)\right]= \\
= & =\left(\frac{\xi}{x}\right)^{1-\alpha-\beta} \frac{M(\xi)}{x-\xi}
\end{aligned}
$$

где $F(a, b ; c ; z)$ - гипергеометрическая функция Гаусса [1, с. 31].

Аналогично

$$
\begin{aligned}
& K_{2}(x, \xi)=M(\xi) \frac{d}{d x} \int_{0}^{x} \frac{d t}{(x-t)^{1-\alpha-\beta}(\xi-t)^{\alpha+\beta}}- \\
& I_{3}(x, \xi)=M(\xi) \frac{d}{d x} \int_{0}^{x} \frac{[M(\xi)-M(t)] d t}{(x-t)^{1-\alpha-\beta}(\xi-t)^{\alpha+\beta}} \\
& (x-t)^{1-\alpha-\beta}(\xi-t)^{\alpha+\beta}
\end{aligned}
$$




$$
=\frac{M(\xi)}{\alpha+\beta} \frac{d}{d x}\left[\left(\frac{x}{\xi}\right)^{\alpha+\beta} F\left(\alpha+\beta, 1 ; 1+\alpha+\beta ; \frac{x}{\xi}\right)\right]=\left(\frac{\xi}{x}\right)^{1-\alpha-\beta} \frac{M(\xi)}{\xi-x} .
$$

Тогда уравнение (14) примет вид

$$
A^{*}(x) \nu(x)+\int_{0}^{1} \frac{K^{*}(x, \xi) \nu(\xi) d \xi}{\xi-x}=F^{*}(x),
$$

где

$$
\begin{gathered}
A^{*}(x)=1+\cos [\pi(\alpha+\beta)] M(x), \\
K^{*}(x, \xi)= \begin{cases}\mu K_{1}(x, \xi)(x-\xi), & \xi \leqslant x, \\
\mu K_{2}(x, \xi)(\xi-x), & \xi \geqslant x,\end{cases} \\
F^{*}(x)=D_{0+}^{1-\alpha-\beta}\left[\frac{F(x)}{A(x)}\right] .
\end{gathered}
$$

Из установленных свойств ядер $K_{1}(x, \xi)$ и $K_{2}(x, \xi)$ заключаем, что ядро $K^{*}(x, \xi)$ дважды непрерывно дифференцируемо в квадрате $0<x, \xi<1$ при $\xi \neq x$ и допускает оценку

$$
K^{*}(x, \xi)=\mathrm{O}(1)(\xi-x)^{-1}
$$

где $\mathrm{O}(1)$ означает ограниченную в $\bar{I} \times \bar{I}$ величину.

В силу условий (2) и свойств дробных производных можно заключить, что $F^{*}(x) \in C^{1}(I)$.

Таким образом, уравнение $(15)$ при $A^{*}(x) \neq 0$ есть сингулярное интегральное уравнение [8, с. 157] с ядром Коши.

Условие

$$
\left[A^{*}(x)\right]^{2}+\pi^{2}\left[K^{*}(x)\right]^{2} \neq 0
$$

гарантирует существование регуляризатора, приводящего уравнение (15) к интегральному уравнению Фредгольма второго рода. Отсюда и из единственности искомого решения следует существование решения исследуемой задачи.

СлучАй 2. Пусть в областях $\Omega_{1}$ и $\Omega_{2}$ выполняются требования (6), (7). Тогда на основании условия сопряжения 2) и соотношений (12) имеем

$$
D_{0+}^{1-\alpha-\beta} \tau(x)+N(x) D_{1-}^{1-\alpha-\beta} \tau(x)+P(x) \tau(x)=Q(x),
$$

где

$$
\begin{gathered}
N(x)=\frac{\widetilde{B}_{1}(x)-\mu(x) \widetilde{B}_{2}(x)}{\widetilde{A}_{1}(x)-\mu(x) \widetilde{A}_{2}(x)}, \quad P(x)=\frac{\widetilde{D}_{1}(x)-\mu(x) \widetilde{D}_{2}(x)}{\widetilde{A}_{1}(x)-\mu(x) \widetilde{A}_{2}(x)}, \\
Q(x)=\frac{\mu(x) \widetilde{\gamma}_{2}(x)+\lambda(x)-\widetilde{\gamma}_{1}(x)}{\widetilde{A}_{1}(x)-\mu(x) \widetilde{A}_{2}(x)}, \quad \widetilde{A}_{1}(x)-\mu(x) \widetilde{A}_{2}(x) \neq 0 .
\end{gathered}
$$

Действуя на обе части оператором $I_{0+}^{1-\alpha-\beta}$ и используя результаты $[4$, с. 98-103], после преобразований получим

$$
\bar{A}^{*}(x) \tau(x)+\int_{0}^{1} \frac{\bar{K}^{*}(x, \xi) \tau(\xi) d \xi}{\xi-x}=\bar{F}^{*}(x),
$$


где

$$
\begin{aligned}
& \bar{A}^{*}(x)=1+\pi \operatorname{ctg}[\pi(\alpha+\beta)] N(x), \quad \bar{F}^{*}(x)=I_{0+}^{1-\alpha-\beta} Q(x), \\
& \bar{K}^{*}(x, \xi)=\left\{\begin{aligned}
\frac{\sin [\pi(\alpha+\beta)]}{\pi}\left[K_{5}(x, \xi)-K_{3}(x, \xi)\right](x-\xi)+ & \\
+\frac{1}{\Gamma(1-\alpha-\beta)} P(\xi)(x-\xi)^{1-\alpha-\beta} & \text { при } \quad x \geqslant \xi, \\
\frac{\sin [\pi(\alpha+\beta)]}{\pi}\left[K_{6}(x, \xi)-K_{4}(x, \xi)\right](\xi-x) & \text { при } \quad x \leqslant \xi,
\end{aligned}\right. \\
& K_{3}(x, \xi)=\int_{0}^{\xi} \frac{N^{\prime}(t) d t}{(x-t)^{\alpha+\beta}(\xi-t)^{1-\alpha-\beta}}, \\
& K_{4}(x, \xi)=\int_{0}^{x} \frac{N^{\prime}(t) d t}{(x-t)^{\alpha+\beta}(\xi-t)^{1-\alpha-\beta}}, \\
& K_{5}(x, \xi)=\frac{d}{d x} \int_{0}^{\xi} \frac{N(t) d t}{(x-t)^{\alpha+\beta}(\xi-t)^{1-\alpha-\beta}}, \\
& K_{6}(x, \xi)=\frac{d}{d x} \int_{0}^{x} \frac{N(t) d t}{(x-t)^{\alpha+\beta}(\xi-t)^{1-\alpha-\beta}} .
\end{aligned}
$$

Теперь достаточно повторить аргументацию доказательства существования решения задачи первого случая.

СлучАй 3. Пусть в области $\Omega_{1}$ выполняется условие $(3)$, а в области $\Omega_{2}-$ условие (10). Краткости ради положим $C_{1}(x)=0, \mu(x)=1, \lambda(x)=0$.

Учитывая условия сопряжения 2), соотношение (11) при $i=1$ и соотношение (12) при $i=2$, получим уравнение

$$
\begin{gathered}
\tau(x)-\bar{A}_{1}(x) I_{0+}^{1-\alpha-\beta} \widetilde{A}_{2}(x) D_{0+}^{1-\alpha-\beta} \tau(x)-\bar{A}_{1}(x) I_{0+}^{1-\alpha-\beta} \widetilde{B}_{2}(x) D_{1-}^{1-\alpha-\beta} \tau(x)- \\
-\bar{B}_{1}(x) I_{1-}^{1-\alpha-\beta} \widetilde{A}_{2}(x) D_{0+}^{1-\alpha-\beta} \tau(x)-\bar{B}_{1}(x) I_{1-}^{1-\alpha-\beta} \widetilde{B}_{2}(x) D_{1-}^{1-\alpha-\beta} \tau(x)- \\
-\bar{A}_{1}(x) I_{0+}^{1-\alpha-\beta} \widetilde{D}_{2}(x) \tau(x)-\bar{B}_{1}(x) I_{1-}^{1-\alpha-\beta} \widetilde{D}_{2}(x) \tau(x)= \\
=\bar{A}_{1}(x) I_{0+}^{1-\alpha-\beta} \widetilde{\gamma}_{2}(x)+\bar{B}_{1}(x) I_{1-}^{1-\alpha-\beta} \widetilde{\gamma}_{2}(x)+\bar{\gamma}_{1}(x) .
\end{gathered}
$$

Преобразуем уравнение (16). Рассмотрим вначале второе слагаемое (без учёта внешнего коэффициента $\left.-\bar{A}_{1}(x)\right)$ :

$$
I_{11}(x)=I_{0+}^{1-\alpha-\beta} \widetilde{B}_{2}(x) D_{1-}^{1-\alpha-\beta} \tau(x) .
$$

Вычисления, проведённые для второго случая, дают возможность записать $I_{11}(x)$ в виде

$$
\begin{aligned}
& I_{11}(x)=\pi \operatorname{ctg}[\pi(\alpha+\beta)] \widetilde{B}_{2}(x) \tau(x)+ \\
& \quad+\frac{\sin [\pi(\alpha+\beta)]}{\pi} \int_{0}^{x}\left[K_{7}(x, \xi)-K_{8}(x, \xi)\right] \tau(\xi) d \xi+ \\
& \quad+\frac{\sin [\pi(\alpha+\beta)]}{\pi} \int_{x}^{1}\left[K_{9}(x, \xi)-K_{10}(x, \xi)\right] \tau(\xi) d \xi,
\end{aligned}
$$


где ядра $K_{7}(x, \xi), \ldots, K_{10}(x, \xi)$ имеют такой же вид, что и ядра $K_{3}(x, \xi), \ldots$, $K_{6}(x, \xi)$, только функцию $N(t)$ надо заменить функцией $\widetilde{B}_{2}(t)$.

Рассмотрим

$$
\begin{aligned}
& I_{12}(x)=I_{0+}^{1-\alpha-\beta} \widetilde{A}_{2}(x) D_{0+}^{1-\alpha-\beta} \tau(x)= \\
& \quad=\frac{\sin [\pi(\alpha+\beta)]}{\pi} \int_{0}^{x} \frac{\widetilde{A}_{2}(t) d t}{(x-t)^{\alpha+\beta}} \frac{d}{d t} \int_{0}^{t} \frac{\tau(\xi) d \xi}{(t-\xi)^{1-\alpha-\beta}} .
\end{aligned}
$$

В силу равенства

$$
\begin{aligned}
I_{13}(x) & =\frac{d}{d x} \int_{0}^{x} \frac{\widetilde{A}_{2}(t) d t}{(x-t)^{\alpha+\beta}} \int_{0}^{t} \frac{\tau(\xi) d \xi}{(t-\xi)^{1-\alpha-\beta}}= \\
& =\int_{0}^{x} \frac{\widetilde{A}_{2}(t) d t}{(x-t)^{\alpha+\beta}} \frac{d}{d t} \int_{0}^{t} \frac{\tau(\xi) d \xi}{(t-\xi)^{1-\alpha-\beta}}+\int_{0}^{x} \frac{\widetilde{A}_{2}^{\prime}(t) d t}{(x-t)^{\alpha+\beta}} \int_{0}^{t} \frac{\tau(\xi) d \xi}{(t-\xi)^{1-\alpha-\beta}}
\end{aligned}
$$

имеем (после перемены порядка интегрирования)

$$
I_{12}(x)=\frac{\sin [\pi(\alpha+\beta)]}{\pi} \int_{0}^{x} K_{11}(x, \xi) \tau(\xi) d \xi
$$

где

$$
K_{11}(x, \xi)=\frac{d}{d x} \int_{\xi}^{x} \frac{\widetilde{A}_{2}(t) d t}{(x-t)^{\alpha+\beta}(t-\xi)^{1-\alpha-\beta}}-\int_{\xi}^{x} \frac{\widetilde{A}_{2}(t) d t}{(x-t)^{\alpha+\beta}(t-\xi)^{1-\alpha-\beta}}
$$

Очевидно, что поведение ядра $K_{11}(x, \xi)$ аналогично поведению в смысле гладкости интегралов

$$
\begin{aligned}
& I_{14}^{(1)}(x)=\widetilde{A}_{2}(x) \frac{d}{d x} \int_{\xi}^{x} \frac{d t}{(x-t)^{\alpha+\beta}(t-\xi)^{1-\alpha-\beta}}= \\
&=\widetilde{A}_{2}(x) \frac{d}{d x} B(\alpha+\beta, 1-\alpha-\beta)=0
\end{aligned}
$$

и

$$
I_{14}^{(2)}(x)=A_{2}^{\prime}(x) B(1+\beta, 1-\alpha-\beta),
$$

где $B(a, b)$ - бета-функция [1, с. 31].

Рассмотрим

$$
\begin{aligned}
& I_{15}(x, \xi)=I_{1-}^{1-\alpha-\beta} \widetilde{A}_{2}(x) D_{0+}^{1-\alpha-\beta} \tau(x)= \\
& \quad=\frac{\sin [\pi(\alpha+\beta)]}{\pi} \int_{x}^{1} \frac{\widetilde{A}_{2}(t) d t}{(t-x)^{\alpha+\beta}} \frac{d}{d t} \int_{0}^{t} \frac{\tau(\xi) d \xi}{(t-\xi)^{1-\alpha-\beta}} .
\end{aligned}
$$

Проводя необходимые вычисления, получим

$$
I_{15}(x, \xi)=\frac{\sin [\pi(\alpha+\beta)]}{\pi}\left[\int_{0}^{x} K_{12}(x, \xi) \tau(\xi) d \xi+\int_{x}^{1} K_{13}(x, \xi) \tau(\xi) d \xi\right]
$$


где

$$
\begin{aligned}
& K_{12}(x, \xi)=\frac{d}{d x} \int_{x}^{1} \frac{\widetilde{A}_{2}(t) d t}{(t-x)^{\alpha+\beta}(t-\xi)^{1-\alpha-\beta}}- \\
&-\int_{x}^{1} \frac{\widetilde{A}_{2}(t) d t}{(t-x)^{\alpha+\beta}(t-\xi)^{1-\alpha-\beta}}+\frac{\widetilde{A}_{2}(1)}{(1-x)^{\alpha+\beta}(1-\xi)^{1-\alpha-\beta}}, \\
& K_{13}(x, \xi)=\frac{d}{d x} \int_{\xi}^{1} \frac{\widetilde{A}_{2}(t) d t}{(t-x)^{\alpha+\beta}(t-\xi)^{1-\alpha-\beta}}- \\
& \quad-\int_{\xi}^{1} \frac{\widetilde{A}_{2}^{\prime}(t) d t}{(t-x)^{\alpha+\beta}(t-\xi)^{1-\alpha-\beta}}+\frac{\widetilde{A}_{2}(1)}{(1-x)^{\alpha+\beta}(1-\xi)^{1-\alpha-\beta}} .
\end{aligned}
$$

Гладкость $K_{12}(x, \xi)$ и $K_{13}(x, \xi)$ будет определяться соответственно гладкостью

$$
\begin{aligned}
& I_{16}(x, \xi)=\frac{d}{d x} \int_{x}^{1} \frac{d t}{(t-x)^{\alpha+\beta}(t-\xi)^{1-\alpha-\beta}}=\left(\frac{1-\xi}{1-x}\right)^{\alpha+\beta} \frac{1}{\xi-x} \\
& I_{17}(x, \xi)=\frac{d}{d x} \int_{\xi}^{1} \frac{d t}{(t-x)^{\alpha+\beta}(t-\xi)^{1-\alpha-\beta}}=\left(\frac{1-\xi}{1-x}\right)^{\alpha+\beta} \frac{1}{\xi-x}
\end{aligned}
$$

Преобразуем интеграл

$$
\begin{aligned}
I_{18}(x, \xi)= & I_{1-}^{1-\alpha-\beta} \widetilde{B}_{2}(x) D_{1-}^{1-\alpha-\beta} \tau(x)= \\
& =-\frac{\sin [\pi(\alpha+\beta)]}{\pi} \int_{x}^{1} \frac{\widetilde{B}_{2}(t) d t}{(t-x)^{\alpha+\beta}} \frac{d}{d t} \int_{t}^{1} \frac{\tau(\xi) d \xi}{(\xi-t)^{1-\alpha-\beta}}= \\
& =\frac{\sin [\pi(\alpha+\beta)]}{\pi} \int_{x}^{1} K_{14}(x, \xi) \tau(\xi) d \xi,
\end{aligned}
$$

где

$$
K_{14}(x, \xi)=\frac{d}{d x} \int_{x}^{\xi} \frac{\widetilde{B}_{2}(t) d t}{(t-x)^{\alpha+\beta}(\xi-t)^{1-\alpha-\beta}}+\int_{x}^{\xi} \frac{\widetilde{B}_{2}^{\prime}(t) d t}{(t-x)^{\alpha+\beta}(\xi-t)^{1-\alpha-\beta}} .
$$

Гладкость ядра $K_{14}(x, \xi)$ будет определяться гладкостью

$$
I_{19}=\frac{d}{d x} \int_{x}^{\xi} \frac{d t}{(t-x)^{\alpha+\beta}(\xi-t)^{1-\alpha-\beta}}=\frac{d}{d x} B(1-\alpha-\beta, \alpha+\beta)=0 .
$$

Следовательно, ядро $K_{14}(x, \xi)$ особенностей не имеет и его гладкость будут определять функции $\widetilde{B}_{2}(x)$ и $\widetilde{B}_{2}^{\prime}(x)$.

Теперь уравнению (16) можно придать вид

$$
\mu(x) \tau(x)+\int_{0}^{1} \frac{K^{* *}(x, \xi) \tau(\xi) d \xi}{\xi-x}=F_{1}^{*}(x),
$$


где

$$
K^{* *}(x, \xi)=\left\{\begin{array}{c}
{\left[\frac { \operatorname { s i n } [ \pi ( \alpha + \beta ) ] } { \pi } \left(K_{11}(x, \xi) \bar{A}_{1}(x)-K_{7}(x, \xi)-K_{8}(x, \xi)-\right.\right.} \\
\left.\left.-B_{1}(x) K_{2}(x, \xi)\right)-\frac{\bar{A}_{1}(x) \widetilde{D}_{2}(\xi)}{\Gamma(1-\alpha-\beta)}\right](x-\xi) \quad \text { при } \quad x \geqslant \xi, \\
{\left[\frac{\sin [\pi(\alpha+\beta)]}{\pi} \bar{B}_{1}(x)\left(K_{13}(x, \xi)-K_{14}(x, \xi)\right)-\right.} \\
\left.-\frac{\bar{B}_{1}(x) \widetilde{D}_{2}(\xi)}{\Gamma(1-\alpha-\beta)}\right](\xi-x) \quad \text { при } \quad \xi \geqslant x,
\end{array}\right.
$$

Условие нормальной разрешимости уравнения (17) имеет вид

$$
\mu^{2}(x)+\pi^{2}\left[K^{* *}(x, x)\right]^{2} \neq 0 .
$$

Проведённые вычисления дают возможность провести далее доказательство существования решения задачи аналогично первому случаю, что затруднений не вызывает.

\section{СПИСОК ЛИТЕРАТУРЫ/ REFERENCES}

1. С. Г. Самко, А. А. Килбас, О. И. Маричев, Интеграль и производные дробного порлдка и некоторые их приложения, Минск: Наука и техника, 1987. 688 с. [S. G. Samko, A. A. Kilbas, O. I. Maritchev, Integraly i proizvodnyye drobnogo poryadka $i$ nekotoryye ikh prilozheniya [Integrals and Derivatives of the Fractional Order and Some of their Applications], Minsk, Nauka i Tekhnika, 1987, 688 pp. (In Russian)]

2. А. М. Нахушев, Задачи со смещением для уравнений в частных производных, М.: Наука, 2006. 287 с. [A. M. Nakhushev, Zadachi so smeshcheniyem dlya uravnenii v chastnykh proizvodnykh [Problems with shifts for partial differential equations], Moscow, Nauka, 2006, 287 pp. (In Russian)]

3. М. М. Смирнов, Вырождающиеся гиперболические уравнения, Минск: Высшая школа, 1977. 158 c. [M. M. Smirnov, Vyrozhdayushchiyesya giperbolicheskiye uravneniya [Degenerate Hyperbolic Equations], Minsk, Vysshaya Shkola, 1977, 158 pp.]

4. С. К. Кумыкова, "Краевая задача со смещением для вырождающегося внутри области гиперболического уравнения" // Дифферени. уравнения, 1980. Т. 16, № 1. С. 93104; S. K. Kumykova, "Boundary-value problem with translation for a hyperbolic equation degenerate in the interior of a region", Differ. Equations, 1980, vol. 16, no. 1, pp. 68-76.

5. О. А. Репин, С. К. Кумыкова, "Нелокальная задача для уравнения смешанного типа третьего порядка с обобщенными операторами дробного интегро-дифференцирования произвольного порядка" // Вестн. Сам. гос. техн. ун-та. Сер. Физ.мат. науки, 2011. № 4(25). C. 25-36. doi: 10.14498/vsgtu1014. [O. A. Repin, S. K. Kumykova, "Nonlocal problem for a equation of mixed type of third order with generalized operators of fractional integro-differentiation of arbitrary order", Vestn. Samar. Gos. Tekhn. Univ. Ser. Fiz.-Mat. Nauki, 2011, no. 4(25), pp. 25-36. (In Russian)].

6. С. К. Кумыкова, Ф. Б. Нахушева, “Об одной краевой задаче для гиперболического уравнения, вырождающегося внутри области” // Дифферени. уравнения, 1978. Т. 14, № 1. C. 50-65; S. K. Kumykova, F. B. Nakhusheva, "A boundary-value problem for a hyperbolic equation degenerate in the interior of a region", Differ. Equations, 1978, vol. 14, no. 1, pp. 3546. 
7. О. А. Репин, Краевые задачи со смещением для уравнений гиперболического и смешанного типов, Самара: Саратов. гос. ун-т, Самарский филиал, 1992. 164 с. [O. A. Repin, Krayevyye zadachi so smeshcheniyem dlya uravneniy giperbolicheskogo $i$ smeshannogo tipov [Boundary value problems with shift for equations of hyperbolic and mixed type], Samara, Saratov State Univ., Samara Branch, 1992, 164 pp. (In Russian)]

8. Н. И. Мусхелишвили, Сингулярные интегралъные уравнения, М.: Наука, 1968. 512 с. [N. I. Muskhelishvili, Singulyarnyye integral'nyye uravneniya [Singular Integral Equations], Moscow, Nauka, 1968, 512 pp. (In Russian)]

Поступила в редакцию 04/XII/2013;

в окончательном варианте - 11/II/2014;

принята в печать $-26 / \mathrm{II} / 2014$.

MSC: 35L80; 35L20, 35C15

\title{
A BOUNDARY-VALUE PROBLEM WITH SHIFT FOR A HYPERBOLIC EQUATION DEGENERATE IN THE INTERIOR OF A REGION
}

\author{
O. A. Repin ${ }^{1,2}$, S. K. Kumykova ${ }^{3}$ \\ 1 Samara State Economic University, \\ 141, Sovetskoy Armii st., Samara, 443090, Russian Federation. \\ 2 Samara State Technical University, \\ 244, Molodogvardeyskaya st., Samara, 443100, Russian Federation. \\ 3 Kabardino-Balkarian State University, \\ 173, Chernyshevskogo st., Nalchik, 360004, Russian Federation.
}

For a degenerate hyperbolic equation in characteristic region (lune) a boundary-value problem with operators of fractional integro-differentiation is studied. The solution of this equation on the characteristics is related point-to-point to the solution and its derivative on the degeneration line. The uniqueness theorem is proved by the modified Tricomi method with inequality-type constraints on the known functions. Question of the problem solution's existence is reduced to the solvability of a singular integral equation with Cauchy kernel of the normal type.

Keywords: Cauchy problem, boundary-value problem with shift, fractional integrodifferentiation operators, singular equation with Cauchy kernel, regularizer, Gauss hypergeometric function, Euler gamma function.

Received 04/XII/2013;

received in revised form $11 / \mathrm{II} / 2014$;

accepted $26 / \mathrm{II} / 2014$.

ISSN: 2310-7081 (online), 1991-8615 (print); doi: http://dx.doi.org/10.14498/vsgtu1280 (C) 2014 Samara State Technical University.

Citation: O. A. Repin, S. K. Kumykova, "A Boundary-value Problem with Shift for a Hyperbolic Equation Degenerate in the Interior of a Region", Vestn. Samar. Gos. Tekhn. Univ., Ser. Fiz.-Mat. Nauki [J. Samara State Tech. Univ., Ser. Phys. \& Math. Sci.], 2014, no. 1 (34), pp. 37-47. doi: 10.14498/vsgtu1280. (In Russian)

Authors Details: Oleg A. Repin (Dr. Phys. \& Math. Sci.), Head of Dept., Dept. of Mathematical Statistics and Econometrics ${ }^{1}$; Professor, Dept. of Applied Mathematics \& Computer Science ${ }^{2}$. Svetlana K. Kumykova (Cand. Phys. \& Math. Sci.), Associate Professor, Dept. of Function Theory.

E-mail addresses: matstat@mail.ru (O.A. Repin, Corresponding author), bsk@rect.kbsu.ru (S.K. Kumykova) 